\title{
Addendum: The function of follicular helper T cells is regulated by the strength of $T$ cell antigen receptor binding
}

\author{
Nicolas Fazilleau, Louise J McHeyzer-Williams, Hugh Rosen \& Michael G McHeyzer-Williams \\ Nat. Immunol. 10, 375-384 (2009); published online 1 March 2009; corrected online 8 March 2009; addendum published after print \\ 8 March 2011
}

\section{Plasma cells negatively regulate the follicular helper T cell program}

\author{
Nadége Pelletier, Louise J McHeyzer-Williams, Kurt A Wong, Eduard Urich, Nicolas Fazilleau \& Michael G McHeyzer-Williams \\ Nat. Immunol. 11, 1110-1118 (2010); published online 31 October 2010; addendum published after print 8 March 2011
}

It has been called to our attention that in a series of studies on the development of follicular helper $\mathrm{T}$ cells ( $\mathrm{T}_{\mathrm{FH}}$ cells), the primers we used to amplify $B c l 6$ were specific for $B c l 6 b$, not Bcl6. We have now traced back the results and have redone experiments that demonstrate that both $B c l 6$ and $B c l 6 b$ are modified in the same way and to a similar extent in antigen-specific and non-antigen-specific $\mathrm{T}_{\mathrm{FH}}$ cells induced both in vivo and in vitro.

In the original studies we inadvertently mixed up primers specific for Bcl6b (but not for Bcl6) with those specific for Bcl6. After closer inspection, we found that this error was made when the wrong set of primers was ordered and labeled "Bcl6 \#2" in our laboratory but the primers were in fact specific for $B c l 6 b$. We used both sets of primers in the original studies of gene expression on antigen-specific $\mathrm{T}_{\mathrm{FH}}$ cells analyzed immediately after isolation (Fig. 3f in Nat. Immunol. 10, 375-384 (2009), and Addendum Fig. 1). Hence, the expression of both Bcl6 and Bcl6b seemed to be three- to fourfold higher in pigeon cytochrome $c$ (PCC)-specific $\mathrm{T}_{\mathrm{FH}}$ cells than in naive $\mathrm{CD} 4^{+}$helper $\mathrm{T}$ cells.

As these genes are structurally related, we were concerned that the primers for Bcl6b may have sufficiently cross-reacted with $B c l 6$ and perhaps amplified the latter gene in $\mathrm{T}$ cells. Thus, we sorted antigen-specific $\mathrm{T}_{\mathrm{FH}}$ cells immediately after isolation and amplified random hexamer-primed cDNA with both sets of primers (Addendum Fig. 2). Both gene products were amplified and produced PCR products of different sizes (the predicted sizes for the different gene products). We then sequenced these products to demonstrate they belonged to the different genes as presented. Thus, both primers amplified the expected PCR products in both naive helper T cells and antigen-specific $\mathrm{T}_{\mathrm{FH}}$ cells.

To explore this issue further, we sorted non-antigen-specific $\mathrm{T}_{\mathrm{FH}}$ cells on the basis of cell surface phenotype and amplified DNA from these cells by RT-PCR without further treatment (Addendum Fig. 3). These experiments also showed that both Bcl6 and Bcl6b had similar basal expression in naive helper $\mathrm{T}$ cells and a similar change of expression in CXCR5 $5^{+} \mathrm{T}_{\mathrm{FH}}$ cells.

In our more recent study on antigen presentation by plasma cells (Nat. Immunol. 11, 1110-1118 (2010)), we reported that plasma cells negatively regulate the $\mathrm{T}_{\mathrm{FH}}$ program in vitro and in vivo. Our conclusions were based on expression of the genes encoding interleukin 21 (IL-21) and Bcl-6, as measured by reverse transcription and quantitative PCR. The original primer mix-up continued in the early phase of these studies. However, the error was sporadic and these more recent studies reported on $B c l 6$ and not $B c l 6 b$, as outlined below (Addendum Table 1).

Hence, we concluded that antigen presentation by plasma cells inhibits the expression of mRNA for IL-21 and Bcl-6 by in vivo-derived, endogenous, antigen-specific $\mathrm{T}_{\mathrm{FH}}$ cells (Fig. 5d, second panel, in Nat. Immunol. 11, 1110-1118 (2010)). Furthermore, the adoptive transfer of antigen-pulsed plasma cells significantly inhibited the expression of mRNA for both IL-21 and Bcl-6 on antigen-specific $\mathrm{T}_{\mathrm{FH}}$ cells (Fig. 7i, in Nat. Immunol. 11, 1110-1118 (2010)) without affecting the expression of mRNA for IL-4 or the transcription factor GATA-3 in vivo. Therefore, this provided evidence of antigen-specific negative regulation of the $\mathrm{T}_{\mathrm{FH}}$ program by plasma cells in vivo.

We have also repeated the in vitro experiments using dendritic cells (DCs) or plasma cells as antigen-presenting cells to evaluate the expression of $B c l 6$ and Bcl6b after in vitro induction of $\mathrm{T}_{\mathrm{FH}}$ cell activity (Addendum Fig. 4). These studies demonstrated equivalent induction of both Bcl6 and Bcl6b in vitro with DCs as antigen-presenting cells and naive helper T cells with transgenic expression of the 5C.C7 $\alpha \beta$ T cell antigen receptor ( $\alpha \beta$ TCR) as antigen-specific helper T cells in these cultures. Consistent with the overall conclusions of our earlier study (Nat. Immunol. 11, 1110-1118 (2010)) plasma cells with antigen did not induce Bcl6 or Bcl6b in 5C.C7 $\alpha \beta$ TCR-transgenic helper T cells in vitro.

We did these new experiments without secondary in vitro transcription or specific synthesis of target cDNA and preamplification by PCR to evaluate the extent of signal to be expected for Bcl6 after in vitro activation of $\mathrm{T}_{\mathrm{FH}}$ cells. The tenfold induction of both Bcl6 and Bcl6b in these studies is more in line with the expected results and the results of other studies using standard culturing conditions to induce $\mathrm{T}_{\mathrm{FH}}$ cell activity. These data are also more in line with the expression of $B c l 6$ and $B c l 6 b$ on antigen-specific $\mathrm{T}_{\mathrm{FH}}$ cells derived in vivo we have reported in all our studies. We believe that the starting cell population, in particular the primary culture stimulus, atypical secondary culture conditions and secondary amplification of the assay all contributed to the more enhanced signals we reported before for Bcl6b (Fig. 5b,c in Nat. Immunol. 11, 1110-1118 (2010)).

In conclusion, we mixed up primers for $B c l 6$ amplification and in parts of our published work inadvertently reported on $B c l 6 b$, not $B c l 6 . B c l 6 b$ is a distinct gene but remains a structurally and functionally related member of the $B c l 6$ gene family. We have now been able to revisit this issue and have demonstrated that both Bcl6 and Bcl6b seemed to be coexpressed to a similar extent in naive helper T cells. Both gene products were similarly enhanced in $\mathrm{T}_{\mathrm{FH}}$ cells that emerged in vivo or were derived in vitro. Furthermore, both genes seemed to be inhibited similarly by antigen presentation by plasma cells. Hence, we believe that this addendum will rectify the mistake made in the initial studies and clarify the coexpression of $B c l 6$ and $B c l 6 b$ in antigen-specific $\mathrm{T}_{\mathrm{FH}}$ cells. 


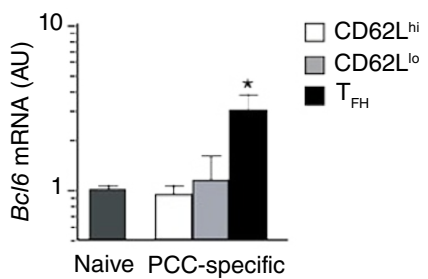

Bc/6 primers

Fwd: CCGGCACGCTAGTGATGTT

Rev:TGTCTTATGGGCTCTAAACTGCT

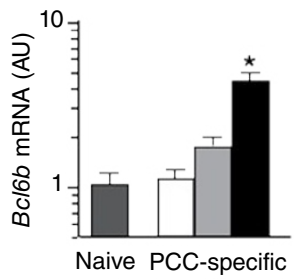

Bc/6b primers

Fwd: GGCTACGTCCGAGAGTTCAC Rev: CTTGTGCGCTCTIAGGGGT
Addendum Figure 1 Upregulation of both $B c / 6$ and $B c / 6 b$ in antigenspecific $T_{F H}$ cells analyzed immediately after isolation. Quantitative PCR analysis of $B c / 6$ mRNA and Bcl6b mRNA in naive helper T cells $\left(\mathrm{V}_{\alpha} 11^{+} \mathrm{V}_{\beta} 3^{+} \mathrm{CD} 44^{\mathrm{l}} \mathrm{CD} 62 \mathrm{~L}^{\text {hi }}\right)$ or PCC-specific $\mathrm{T}_{\mathrm{FH}}$ cells $\left(\mathrm{Pl} \mathrm{I}^{-} \mathrm{B} 22 \mathrm{O}^{-} \mathrm{CD} 11 \mathrm{~b}^{-}\right.$ CD8 $\left.\mathrm{V}_{\alpha} 11^{+} \mathrm{V}_{\beta} 3^{+} \mathrm{CD} 44^{\text {hi }} \mathrm{CD} 62 \mathrm{~L}^{\text {lo }} \mathrm{CXCR} 5^{\text {hi }}\right)$ sorted from draining lymph nodes on day 7 after subcutaneous priming with PCC in Ribi adjuvant $\left(2 \times 10^{3}\right.$ cells), followed by cDNA synthesis and amplification with Platinum SYBR Green (details as for Figs. 1-3 in the original study (Nat. Immunol. 10, 375-384 (2009))); results are presented in arbitrary units (AU) relative to the expression of $\beta_{2}$-microglobulin mRNA, set as 1 . Other antigen-specific helper T cell subsets are sorted on the basis of differences in the expression of $\mathrm{CD} 62 \mathrm{~L}$ and $\mathrm{CXCR} 5$ as described in detail in the original study ( $\mathrm{Nat}$. Immunol. 10, 375-384 (2009)).

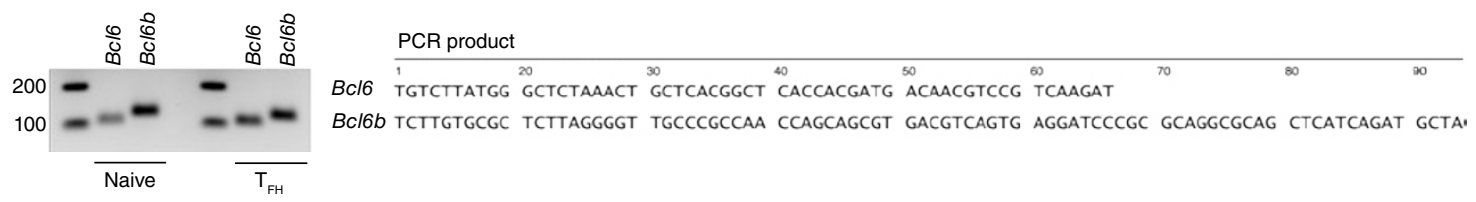

Addendum Figure 2 Primers for Bcl6 and Bcl6b amplify the expected PCR products from naive helper T cells and $T_{F H}$ cells. PCR analysis of Bcl6 and $B c / 6 b$ in naive helper T cells or antigen-specific $\mathrm{T}_{\mathrm{FH}}$ cells $\left(5 \times 10^{3}\right)$ sorted (as described in Addendum Fig. 1 ) on day 8 of the memory response to PCC; cDNA synthesized from RNA by random-hexamer priming was analyzed by 40 cycles of PCR with both sets of primers (Addendum Fig. 1), then primers were removed and PCR products were analyzed directly with a BigDye Terminator Cycle Sequencing kit on a 3130 Genetic Analyzer.
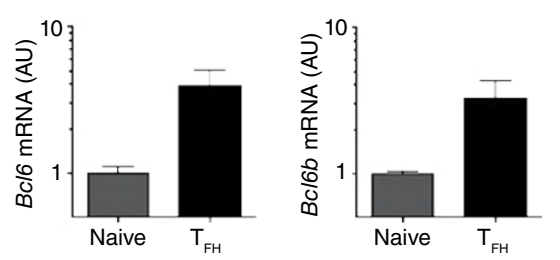

Addendum Figure 3 Equivalent upregulation of $B c / 6$ and $B c / 6 b$ in $C X C R 5^{+} T_{F H}$ cells assessed immediately after isolation. Reverse transcription and quantitative PCR analysis of $B c / 6$ and $B c / 6 b$ in naive helper $\mathrm{T}$ cells $\left(\mathrm{PI}^{-} \mathrm{CD} 8{ }^{-} \mathrm{CD} 11 \mathrm{~b}^{-} \mathrm{CD} 4{ }^{+} \mathrm{CD} 44^{\mathrm{lo}} \mathrm{CD} 62 \mathrm{~L}^{\mathrm{hi}} \mathrm{CXCR} 5^{-}\right)$or $\mathrm{T}_{\mathrm{FH}}$ cells $\left(\mathrm{PI}^{-} \mathrm{CD} 8^{-} \mathrm{CD} 11 \mathrm{~b}^{-}\right.$ $\mathrm{CD} 4{ }^{+} \mathrm{CD} 44^{\text {hi }} \mathrm{CD} 62 \mathrm{~L}^{\text {lo }} \mathrm{CXCR} 5^{+}$) from the draining lymph nodes of mice immunized with Ribi adjuvant only and sorted, results are presented in arbitrary units relative to the expression of $\beta$-actin mRNA.
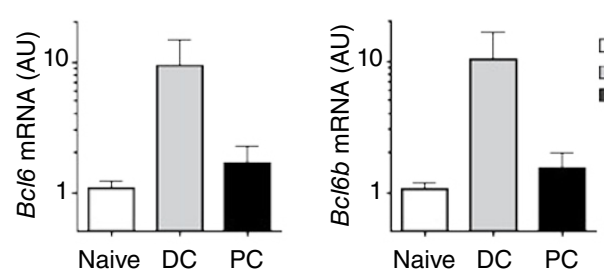

\begin{abstract}
Addendum Figure 4 Selective upregulation of $B c / 6$ and $B c / 6 b$ in antigenspecific $T_{F H}$ cells with the use of DCs but not PCs for antigen presentation in vitro. Quantitative PCR analysis of $B c / 6$ and $B c / 6 b$ in cells derived from $\mathrm{CD} 11 \mathrm{c}^{+} \mathrm{DCs}$ or $\mathrm{IgM}^{-} \mathrm{CD} 138^{+}$plasma cells $(\mathrm{PC})$ sorted ex vivo with protein antigen and cultured for $4 \mathrm{~d}$ in vitro together with cytosolic dye CFSE-labeled naive CD4+ PCC-specific 5C.C7 $\alpha \beta$ TCR-transgenic helper T cells, followed by sorting of CD44hi 5C.C7 $\alpha \beta$ TCR-transgenic helper T cells that diluted CFSE, for RNA extraction, cDNA synthesis and SYBR Green-based quantitative PCR amplification of $B c / 6$ and $B c / 6 b$ (details as for Fig. 4 in the original study (Nat. Immunol. 11, 1110-1118 (2010))); results are presented in arbitrary units relative to $\beta_{2}$-microglobulin mRNA expression.
\end{abstract}

Addendum Table 1 Induction and inhibition of $B c / 6$ or $B c / 6 b$ in $\mathrm{T}_{\mathrm{FH}}$ cells

\begin{tabular}{cccc}
\hline Figure & Amplification method & $\mathrm{T}_{\mathrm{m}}$ & Gene amplified \\
\hline $4 \mathrm{c}$ & $\mathrm{IVT}$ & $86.88-87.32$ & $B c / 6 b$ \\
$5 \mathrm{~b}$ & None & $87.02-87.31$ & $B c / 6 b$ \\
$5 \mathrm{c}$ & STA & $87.03-87.47$ & $B c / 6 b$ \\
$5 \mathrm{~d}$ & STA & $82.75-83.06$ & $B c / 6$ \\
$7 \mathrm{i}$ & STA & $82.99-83.56$ & $B c / 6$ \\
\hline
\end{tabular}

Induction of $B c / 6$ or $B c / 6 b$ in $T_{F H}$ cells selectively by $D C$ antigen presentation and inhibition of $B c / 6$ by PC antigen presentation, assessed by two rounds of in vitro amplification (IVT) of RNA with a MessengerAmp II aRNA Amplification kit (Ambion), similar to what is done before microarray analysis, followed by the generation of CDNA from $10 \mu \mathrm{g}$ amplified RNA as the starting material for SYBR Green-based quantitative PCR analysis; by specific target amplification (STA) with specific primers to generate cDNA, followed by 14 cycles of PCR preamplification and dilution of the product 1:5 for use as the starting material for SYBR Green-based quantitative PCR analysis; or the melting curve $\left(T_{m}\right)$ peak signal after SYBR Green-based quantitative PCR analysis. 